\title{
Design Procedure for New Compact Waffle-Iron Filters with Transmission Zeros
}

\author{
Fernando Teberio, Jon M. Percaz, Ivan Arregui, Petronilo Martin-Iglesias, \\ Txema Lopetegi, Miguel A. G. Laso, and Israel Arnedo
}

\begin{abstract}
In this paper, a novel waffle-iron filter with transmission zeros at multiple frequencies, along with its design procedure, is presented. The proposed filter features a high-power behavior and a wide rejected band in a single compact structure by means of a set of transmission zeros that can also be placed close to the passband. Its design method rests on a divide-and-rule strategy, where the physical dimensions of the constituent design entities (DEs) can be easily computed in a very short time. A novel high-power compact waffle-iron filter with transmission zeros at multiple frequencies has been designed as well as several classical waffle-iron filters with transmission zeros at one frequency only, using a detailed step-by-step procedure which avoids the bruteforce optimizations needed until now. Multipactor and corona simulations have been conducted proving a high-power handling capability of $1.8 \mathrm{~kW}$ and $78.6 \mathrm{~W}$, respectively. A prototype of the novel filter has been fabricated, obtaining a remarkable accordance between the simulated and measured results.
\end{abstract}

Index Terms - Doubly corrugated, high-power, low-pass filter, microwave filters, waffle-iron.

\section{INTRODUCTION}

$\mathrm{T}$ EE classical waffle-iron filter is the typical waveguide lowpass solution when the suppression of the fundamental and the higher-order modes are required over a wide stopband (up to several harmonics). Invented in the 1940s [1], [2], this kind of rectangular waveguide filters is nowadays utilized in many widespread applications such as: antenna feeders [3], [4], output section of a satellite communication payload [5], [6], industrial fabrication processes employing microwaves in order to prevent the escape of radiation from the manufacturing chamber [7], [8], etcetera.

The physical structure of a waffle-iron filter is based on the classical corrugated low-pass filter [9], [10], where longitudinal slots are added to suppress the higher-order $\mathrm{TE}_{\mathrm{n} 0}$ modes over the required stopband. The effect of the slots is very well explained in modern textbooks such as [11] and it can be summarized as follows: due to the fact that any $\mathrm{TE}_{\mathrm{n} 0}$ mode can be decomposed in two plane waves propagating at different angles, if the waffle-iron filter is designed roughly isotropic

This paper is an expanded version from the 2018 International Microwave Symposium, Philadelphia, PA, USA, 10-15 June 2018

This work was supported by ESA's Networking/Partnering Initiative (NPI) under contract no. 4000114859/15/NL/HK and MINECO (Spain) under projects TEC2017-85529-C3-2-R, TEC2014-55735-C3-R, and TEC201451902-C2-2-R (having the same characteristics at a given frequency for any plane wave propagating in any direction), all these plane waves will be similarly attenuated. Thus, in a doubly-corrugated waffle-iron filter all the $\mathrm{TE}_{\mathrm{n} 0}$ modes will be properly suppressed. There are two classical synthesis procedures to design waffle-iron filters [10]. The first one starts designing a classical corrugated waveguide filter. Then, longitudinal slots are incorporated in the low-height sections. This decreases their capacitance per unit length, which makes necessary to reduce further those heights using the tables in [1] in order to maintain the performance of the original structure. The second procedure proposes a new electrical model that includes the E-plane Tjunction available in Marcuvitz's book [12] to predict the frequency behavior of the waffle-iron section. Both methods are rather complicated procedures that may be difficult to control, specifically to obtain the intended in-band return loss of the filter [13]. However, they are still used today to obtain an initial structure whose physical dimensions will be later on intensively optimized (using different numerical analysis methods) to meet the desired frequency specifications [7], [14], [15]. In [16], the authors presented a procedure for the design of classical waffleiron filters that is accurate when the maximum height of the filtering structure is small enough to avoid the excitation of the higher-order non- $\mathrm{TE}_{\mathrm{n} 0}$ modes inside the device up to the maximum frequency of the intended stopband. This assumption allows the design of waffle-iron filters with a limited powerhandling capability only (multipactor threshold levels around hundreds of Watts at $\mathrm{Ku}$ band).

The high-power performance of a waffle-iron filter can be improved by modifying the geometry of its teeth [17], by stacking several identical waffle-iron filters, dividing the power between them and combining their outputs [18] or by enlarging the minimum mechanical gap [19]. Applying the latter approach, if the maximum height is not altered, either the attenuation level along with the rejection bandwidth are reduced or the electrical model is not satisfied. Thus, the maximum height needs to be increased, which leads to the appearance of a transmission zero within the rejection band. However, when this transmission zero is put close to the passband (to increase its steepness for instance), the rejection

Fernando Teberio, Jon M. Percaz, Ivan Arregui, Txema Lopetegi, Miguel A. G. Laso, and Israel Arnedo are with the Electrical and Electronic Engineering Department, Public University of Navarre, Pamplona E-31006, Spain (e-mail: israel.arnedo@unavarra.es).

Petronilo Martin-Iglesias is with the European Space Agency ESA-ESTEC, Noordwijk, The Netherlands. 
bandwidth is also severely decreased. Therefore, there is always a trade-off between the mechanical gap of the structure (i.e., its power-handling capability) and the maximum frequency of the stopband (i.e., its rejection bandwidth). This limitation could be surpassed by connecting several waffle-iron filters with larger gaps whose narrow stopbands are sequentially overlapped to achieve the required wide stopband. However, each waffle-iron filter would have to be independently designed and then connected to each other by quarter-wave transformers, which is time-consuming and leads to a bulky solution [6], [19].

In this paper, we expand [16] and the current state-of-the-art of waffle-iron filters in two different ways. Firstly, the new technique of [16] is extended to include the design of highpower waffle-iron filters (thousands of Watts at $\mathrm{Ku}$ band for multipactor) with transmission zeros at one frequency, as those used in [19]. Secondly, a new waffle-iron structure that provides very compact high-power wide-rejection filters with transmission zeros at multiple frequencies is also presented along with its design procedure.

After this Introduction, the step-by-step design procedure is formally detailed in Section II. In Section III.A, three classical waffle-iron filters are designed with transmission zeros at a single frequency (placed at different locations within the stopband in each example), demonstrating the accuracy of the design procedure, while in Section III.B a novel compact highpower waffle-iron filter with transmission zeros at different frequencies has been designed and fabricated by milling. The paper ends with the concluding remarks of Section IV.

\section{Design MethoD}

In this Section, a complete step-by-step design procedure is proposed of a new structure that alternates rectangular waveguide sections and waffle-iron sections (i.e., rectangular waveguide sections with longitudinal slots) of different heights, see Fig. 1. This novel structure provides higher degrees of freedom than the classical one and, therefore, more design flexibility. However, this comes along with a higher number of

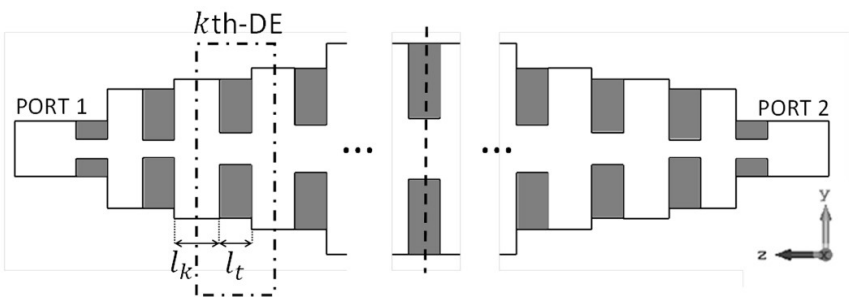

(a)

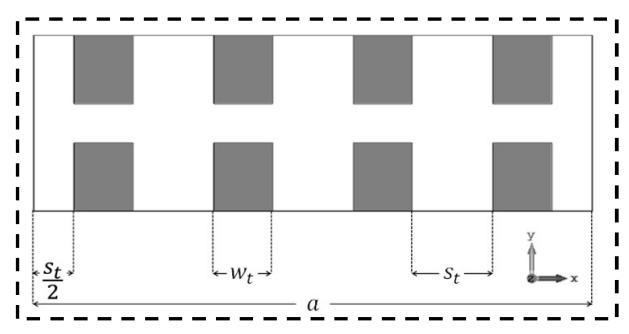

(b)

Fig. 1. (a) Longitudinal view of the entire waffle-iron structure. (b) Transversal view of the waffle-iron section with labelled design parameters.

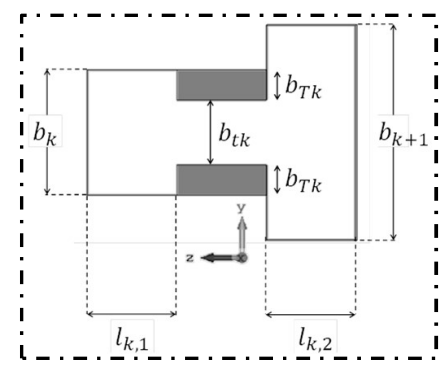

(a)

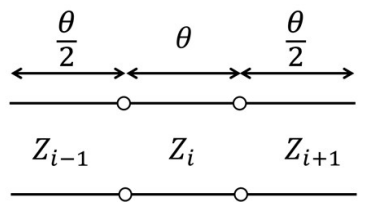

(b)

Fig. 2. (a) Longitudinal view of the $k$ th-DE with its associated constituent parameters. (b) Electrical model of the DE.

design variables and, consequently, it makes unsuccessful typical brute-force optimization techniques.

Notice how the filter in Fig. 1 is divided into $N_{D E}$ Design Entities (DEs), which are obtained independently and connected afterwards. Each DE is defined as two half sections of uniform rectangular waveguide with a waffle-iron section between them (see Fig. 2(a)). As the first step in our method, the low-pass stepped-impedance prototype, based on a set of $\left(2 \cdot N_{D E}+1\right)$ transmission lines with characteristic impedances $Z_{i}$ and electrical length $\theta$, is computed by means of the Richards' transformation and the Unit Element extraction procedure [20], once the aimed all-pole frequency response, the width of the structure, $a$, the required in-band return loss, the frequency of maximum rejection, $f_{0}$, and the maximum frequency of the passband, $f_{c}$, are fixed. The simplest electrical model of each DE is composed of half transmission line, one step, one transmission line, another step, and, finally, another half transmission line, as shown in Fig. 2(b). Therefore, the $Z_{i}$ 's of the low-pass stepped-impedance prototype are combined in triplets to calculate the frequency response of the electrical model of each DE (for $k=1,2, \ldots, N_{D E}$ ) in reflection and transmission by evaluating (1) and (2), respectively:

$$
\begin{aligned}
& S_{11, k}(f)=\frac{E_{11, k}+\frac{E_{12, k}}{Z_{2 k}}-E_{21, k} \cdot Z_{2 k-2}-E_{22, k} \cdot \frac{Z_{2 k-2}}{Z_{2 k}}}{E_{11, k}+\frac{E_{12, k}}{Z_{2 k}}+E_{21, k} \cdot Z_{2 k-2}+E_{22, k} \cdot \frac{Z_{2 k-2}}{Z_{2 k}}} \\
& \text { for } \quad k=1,2, \ldots, N_{D E} \\
& S_{21, k}(f)=\frac{2 \cdot \sqrt{\frac{Z_{2 k-2}}{Z_{2 k}}}}{E_{11, k}+\frac{E_{12, k}}{Z_{2 k}}+E_{21, k} \cdot Z_{2 k-2}+E_{22, k} \cdot \frac{Z_{2 k-2}}{Z_{2 k}}}
\end{aligned}
$$




$$
\text { for } k=1,2, \ldots, N_{D E}
$$

where $\left[E_{k}\right]=\left[\begin{array}{ll}E_{11, k} & E_{12, k} \\ E_{21, k} & E_{22, k}\end{array}\right]$ is the 2-by-2 transmission ABCD matrix of the $k$ th-DE, calculated following (3) to (5).

$$
\begin{gathered}
{\left[E_{k}\right]=\left[R_{2 k-2}\right] \cdot\left[W_{2 k-1}\right] \cdot\left[R_{2 k}\right]} \\
{\left[R_{i}\right]=\left[\begin{array}{cc}
\cos \left(\frac{\theta}{2}\right) & j \cdot Z_{i} \cdot \sin \left(\frac{\theta}{2}\right) \\
\frac{j}{Z_{i}} \cdot \sin \left(\frac{\theta}{2}\right) & \cos \left(\frac{\theta}{2}\right)
\end{array}\right]} \\
{\left[W_{i}\right]=\left[\begin{array}{cc}
\cos (\theta) & j \cdot Z_{i} \cdot \sin (\theta) \\
\frac{j}{Z_{i}} \cdot \sin (\theta) & \cos (\theta)
\end{array}\right]} \\
\text { for } \quad \begin{array}{c}
i=0,1, \ldots,\left(2 \cdot N_{D E}\right) \\
\theta=\frac{\beta_{10, f}}{\beta_{10, f_{0}}} \cdot \frac{\pi}{2}
\end{array}
\end{gathered}
$$

It is important to note that there is a frequency dependence of $\left[E_{k}\right]$ through the propagation constant of the fundamental TE 10 mode, $\beta_{10, f}$.

After that, for each DE, we calculate the magnitude of (1) evaluated at $f_{c},\left|S_{11, k}\left(f_{c}\right)\right|$, the phase of (1) evaluated at $f_{c}$, $\phi_{k, S_{11}}\left(f_{c}\right)$, and the phase of the $S_{21}(f)$ at $f_{0}, \phi_{k, S_{21}}\left(f_{0}\right)$.

The design method continues by defining several physical parameters of the waffle-iron sections, following the typical well-known considerations for this kind of filters that appear in [10]. Particularly, we define the number of teeth, $n_{t}$, which will be higher for wider stopbands, as well as the length of the teeth, $l_{t}$, their width, $w_{t}$, and their separation, $s_{t}$, which will be chosen equal (or very similar) and large enough to allow, simultaneously, an easy manufacturing and a good isotropy, providing the same characteristics, at a given frequency, for the TEM waves propagating in any direction.

Then, the heights $b_{k}$ (see Fig. 2(a)) are defined. Their values depend on the type of waffle-iron filter selected by the designer, which may be one of the following:

a) a waffle-iron filter with transmission zeros at a single frequency (see Section II.A and Fig. 3(a));

b) a novel waffle-iron filter with transmission zeros at multiple frequencies (see Section II.B and Fig. 3(b)).

At this stage in the design, it is possible to calculate the rest of the physical dimensions of each DE: the height of the teeth, $b_{T k}$, the length of the input rectangular waveguide section, $l_{k, 1}$, and the length of the output rectangular waveguide section, $l_{k, 2}$ (clearly identified in Fig. 2(a)). This is done by carrying out simple $S$-parameter EM (electromagnetic) simulations of each

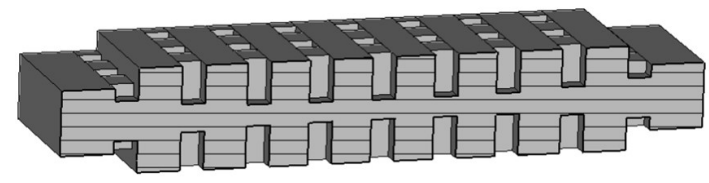

(a)

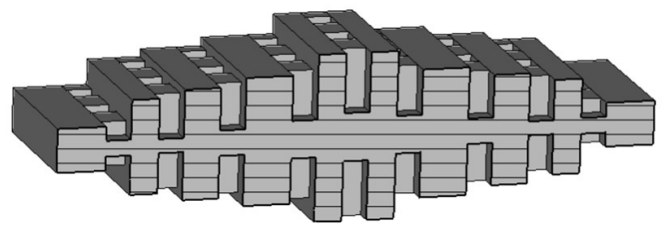

(b)

Fig. 3. 3D cut-view of the (a) waffle-iron filter with transmission zeros at one frequency, and (b) novel waffle-iron filter with transmission zeros at multiple frequencies.

DE individually in order to adjust its frequency response to the one given by its electrical model by modifying $b_{T k}, l_{k, 1}$, and $l_{k, 2}$ in each DE individually. Specifically, $b_{T k}, l_{k, 1}$, and $l_{k, 2}$ are tuned until (6), (7), and (8) are satisfied for each DE (for $k=$ $\left.1,2, \ldots, N_{D E}\right)$ :

$$
\begin{gathered}
\left|S^{\prime}{ }_{11, k}\left(f_{c}\right)\right|=\left|S_{11, k}\left(f_{c}\right)\right| \text { for } k=1,2, \ldots, N_{D E} \\
\phi^{\prime}{ }_{k, S_{11}}\left(f_{c}\right)=\phi_{k, S_{11}}\left(f_{c}\right) \text { for } k=1,2, \ldots, N_{D E} \\
\phi^{\prime}{ }_{k, S_{21}}\left(f_{0}\right)=180^{\circ} \text { for } k=1,2, \ldots, N_{D E}
\end{gathered}
$$

where $\left|S^{\prime}{ }_{11, k}\left(f_{c}\right)\right|$ is the magnitude of $S_{11}(f)$ at $f_{c}, \phi_{k, S_{11}}^{\prime}\left(f_{c}\right)$ is the phase of $S_{11}(f)$ at $f_{c}$, and $\phi^{\prime}{ }_{k, S_{21}}\left(f_{0}\right)$ is the phase of the $S_{21}(f)$ at $f_{0}$ for the $k$ th-DE obtained with an EM simulator. Notice that examining (2), (3), (4) and (5) along with Fig. 2(b), it is obvious that $\phi_{k, S_{21}}\left(f_{0}\right)=2 \cdot \theta_{0}=180^{\circ}$

Finally, the lengths of the different sections, $l_{k}$, are simply computed by (9).

$$
l_{k}=l_{k-1,2}+l_{k, 1} \quad \text { for } \quad k=2,3, \ldots,\left(N_{D E}-1\right)
$$

Moreover, for simplicity, the first, $l_{1}$, and the last, $l_{N_{D E}}$, section lengths will be equal to $l_{1,1}$ (notice that $l_{1,1}$ refers to the length of the first uniform transmission line that affects, as is well known, only to the phase of the frequency response of the entire structure).

It should be noted that although $l_{t}$ has been fixed at the beginning of the design process and it has been kept constant in all DEs, it could be deduced from (3), (4), and (5) that the value of $l_{t}$ is to some extent connected with $\theta$ in the central transmission line in Fig. 2(b). Thus, if (8) is not easily met, the value of $l_{t}$ should be accordingly modified (typically reduced) and $b_{T k}, l_{k, 1}$, and $l_{k, 2}$ recomputed, using again (6) to (8). Besides, it is clear from inspection of Fig. 2(a) that the variables $b_{k, 1}, b_{T k}$ and $b_{t k}$ are related as follows: 


$$
b_{t k}=b_{k}-2 \cdot b_{T k}
$$

Finally, sometimes, a slight adjustment is needed in order to obtain the final frequency response of the required filter, mainly due to the effect of high-order modes that are evanescently present at the intersections of the DEs. However, this is very easily and quickly done by, for instance, the FEST3D Simplex optimization algorithm tool.

\section{A. Waffle-Iron Filter with Transmission Zeros at One Frequency}

The overall filtering structure (see Fig. 3(a)) is composed of the first DE with $b_{1}<b_{2}$ and the last one with $b_{N_{D E}-1}>b_{N_{D E}}$, while the rest of the structure is composed of DEs with $b_{k}=$ $b_{2}=b_{\left(N_{D E}-1\right)}$ for $k=2,3, \ldots,\left(N_{D E}-1\right)$. For the sake of simplicity, a symmetrical end-to-end structure is assumed.

First, the heights of the input and output ports, $b_{1}$ and $b_{N_{D E}}$, respectively, are set to a value lower than $b_{\max }$, given by (11), to avoid the excitation of the higher-order non- $\mathrm{TE}_{\mathrm{n} 0}$ modes through the structure (the $\mathrm{TE}_{01}$ mode in our case) at a selected maximum frequency, $f_{\text {max }}$.

$$
b_{\max }=\frac{c}{2 \cdot f_{\max }}
$$

In order to maximize the high-power handling capability and the stopband attenuation of the structure in [16], several transmission zeros placed at the same frequency (denoted as $f_{Z}$ ) in the stopband can be obtained by setting $b_{k} / 2$ around quarterwavelength at $f_{Z}$, following (12), [19].

$$
b_{k} \gtrsim \frac{c}{2 \cdot f_{Z}}
$$

Therefore, $b_{k}$ for $k=2,3, \ldots,\left(N_{D E}-1\right)$ are estimated choosing the frequency at which the transmission zeros are desired. Usually $f_{Z}=f_{0}$, since it maximizes the attenuation bandwidth and the power handling. However, if a frequency below $f_{0}$ is chosen, the stopband of the filter obtained will be narrower. On the other hand, if a frequency above $f_{0}$ is chosen, the transition slope between the passband and stopband is not improved and the rejection bandwidth hardly increases [19]. Note that if a frequency higher than $f_{\text {max }}$ is chosen, a waffleiron filter without transmission zeros in the stopband will be designed, being a particular case of this paper which has been already discussed in [16]. Hence, a wide stopband and a transmission zero close to the passband cannot be obtained simultaneously. Lastly, it is also important to notice that the actual location of $f_{Z}$ depends also on the physical parameters $b_{t k}, w_{t}$, and $b_{T k}$, [7], [12].

Once $b_{k}$ is estimated by means of (12), the value of $b_{T k}$ (and hence $b_{t k}$ by (10)) is computed by satisfying (6) for the kth-DE with the maximum value of $\left|S_{11, k}\left(f_{c}\right)\right|$, since it will determine the minimum $b_{t k}$ of the filter. Then, if $b_{t k}$ does not fulfil the minimum value required by the designer, $b_{k}$ should be increased by means of reducing $f_{Z}$. However, it must be taken into account that $b_{t k}$ must be small enough to shift the cutoff frequency of the higher-order non- $\mathrm{TE}_{\mathrm{n} 0}$ modes beyond $f_{\max }$. This also minimizes the excitation of the higher-order modes in the inner part of each DE (since higher values of $b_{t k}$ imply higher values of $b_{k}$ and lower values of $l_{k, 1}$ and $l_{k, 2}$ through a capacitive effect - phase shift contribution -). Then, $b_{k}$ and $b_{T k}$ are recalculated to fulfil (6) and the desired $b_{t k}$ (with the previous constraints) simultaneously, considering only the kthDE that has the maximum value of $\left|S_{11, k}\left(f_{c}\right)\right|$. Next, once the final value of $b_{k}$ has been fixed (and being equal for all $k=$ $\left.2,3, \ldots,\left(N_{D E}-1\right)\right)$, the $b_{T k}$ of each DE is calculated to satisfy (6). At the same time, $l_{k, 1}$ and $l_{k, 2}$ of each DE will be calculated by satisfying (7) and (8). Finally, the length of each section, $l_{k}$, is straightforwardly computed by using (9).

In this case, as it will be exemplified in Section III, the synthesis procedure is more accurate if the transmission zeros are closer to $f_{\text {max }}$, since there will be less high-order modes energy contribution in the inner part of the device.

\section{B. Novel Waffle-Iron Filter with Transmission Zeros at Multiple Frequencies}

The novel filter, see Fig. 3(b), is obtained by cascading DEs with different $b_{k}$, which implement transmission zeros located at different frequencies. Physically, the structure begins with the DE which provides the transmission zero located farthest from the passband. Then, the rest of DEs are cascaded in such a way that the $\mathrm{DE}$ that provides the transmission zero closest to the passband will be placed in the center of the device.

The design method starts by designing the DE that provides the transmission zero, $f_{Z, k}$, closest to the passband $\left(k=N_{D E} / 2\right.$ if $N_{D E}$ even or $k=\left(N_{D E}+1\right) / 2$ if $N_{D E}$ odd). Therefore, this $b_{k}$ is computed following (12). After that, the value of $b_{T k}$ is directly calculated using (6) and then $b_{t k}$ is calculated by (10). If $b_{t k}$ is not high enough for an adequate power handling, a higher value should be imposed to provide higher-power handling but small enough to achieve the suppression of the higher-order non- $\mathrm{TE}_{\mathrm{n} 0}$ modes beyond $f_{\max }$ as well as to minimize the excitation of higher-order modes due to the closeness between the waffle-iron sections. Thus, $b_{k}$ and $b_{T k}$ are readjusted to satisfy (6). After that, $l_{k, 1}$, and $l_{k, 2}$ are calculated by fulfilling (7) and (8). If $b_{k}$ is high, it maximizes the high-power behavior but produces narrow stopbands. Indeed, the maximum rejection frequency of each DE, $f_{M, k}$, can be easily computed by the out-of-band EM simulation of the $k$ th-DE.

Subsequently, the design process continues by designing the contiguous DE on the left (reducing $k$ in one unit), which corresponds to $k=N_{D E} / 2-1$ if $N_{D E}$ is even or $k=$ $\left(N_{D E}-1\right) / 2$ if $N_{D E}$ is odd. Note then that $b_{k+1}$ was already computed and, hence, $b_{k}$ for this DE is calculated by (12) to achieve a transmission zero around the frequency $f_{M, k+1}$, which can be selected by the designer satisfying $f_{Z, k} \geq f_{Z, k+1}$. Then, the value of $b_{T k}$ is computed satisfying (6) and the value of $b_{t k}$ calculated by (10). Then, following the same criteria as in the previous DE, if $b_{t k}$ is not the desired one, it can be modified (with the same limitations previously explained) and $b_{k}$ and $b_{T k}$ will be recalculated fulfilling (6). After that, $l_{k, 1}$, and $l_{k, 2}$ are 
computed by satisfying (7) and (8). Reducing again $k$ in 1 unit and proceeding iteratively in this way, the physical parameters of each DE are obtained as in the previous case to achieve the required transmission zero at $f_{Z, k}$ fulfilling the target magnitude value using (6), and the corresponding phases by (7) and (8). It is important to note that the attenuation given by the transmission zeros can be reinforced if several identical DEs are chosen. The last DE to be designed is the one situated at the input port of the structure $(k=1)$. In this case, $b_{1}$ will be set smaller than the value given by $b_{\max }$ through (11) to guarantee the suppression of the higher-order non-TE $\mathrm{E}_{\mathrm{n} 0}$ modes up to $f_{\text {max }}$. Finally, the DEs on the right side of the devices will have the same physical parameters if we assume end-to-end symmetry for the sake of simplicity.

The design procedure can be summarized as the following step-by-step flowchart, which is given to the reader for an automatic implementation using a computer:

Step 1) Define the all-pole frequency response, $N_{D E}, a, f_{0}$, $f_{c}$, and the required return loss.

Step 2) Compute $\theta_{c}$ by using (5) and $Z_{i}$ with [20].

Step 3) Electrical model calculations:

3.1) With $Z_{0}, Z_{1}$, and $Z_{2}$, compute $S_{11,1}\left(f_{c}\right)$ by means of (1) $(k=1)$.

3.2) With $Z_{2}, Z_{3}$, and $Z_{4}$, compute $S_{11,2}\left(f_{c}\right)$ by means of (1) $(k=2)$.

3.3) Proceed for all $Z_{i}$ 's triplets in the same manner to have the rest of $S_{11, k}\left(f_{c}\right)$.

3.4) Evaluate $\left|S_{11, k}\left(f_{c}\right)\right|$ and $\phi_{k, S_{11}}\left(f_{c}\right)$.

Step 4) Define $n_{t}, w_{t}, s_{t}$, and $l_{t}$.

Go to step 5) or 6) depending on your need to have a waffleiron filter with transmission zeros at one frequency 5) or at multiple frequencies 6).

Step 5) Waffle-iron filter with transmission zeros at one frequency (Section II.A):

5.1) Define $f_{\max }$ and calculate $b_{1}$ following (11).

5.2) Define $f_{z}$.

5.3) Define $b_{k}$ following (12), which will be equal for $k=$ $2,3, \ldots,\left(N_{D E}-1\right)$.

5.4) Obtain $b_{T k}$ with EM simulations of each DE to satisfy (6).

5.5) Calculate $b_{t k}$ given by (10). If any $b_{t k}$ is too small, go back to step 5.2) and decrease $f_{z}$.

5.6) Obtain $l_{k, 1}$ and $l_{k, 2}$ with EM simulations of each DE to satisfy (7) and (8).

5.7) Calculate all $l_{k}$ 's with (9).

At this point, all the physical dimensions of the waffle-iron filter with transmission zeros at one frequency have been calculated.

Step 6) Novel Waffle-Iron Filter with Transmission Zeros at Multiple Frequencies (Section II.B):
6.1) Start with $k=N_{D E} / 2$ (if $N_{D E}$ even) or $k=$ $\left(N_{D E}+1\right) / 2$ (if $N_{D E}$ odd)

6.2) Define $f_{Z, k}$ (first transmission zero closest to the passband).

6.3) Define $b_{k}$ following (12).

6.4) Obtain $b_{T k}$ with EM simulations to satisfy (6).

6.5) Calculate $b_{t k}$ given by (10). If $b_{t k}$ is not enough, increase $b_{k}$ and go back to step 6.2).

6.6) Obtain $l_{k, 1}$ and $l_{k, 2}$ with EM simulations to satisfy (7) and (8).

6.7) Reduce $k$ in one unit, define $f_{z, k}\left(\geq f_{z, k+1}\right)$, and repeat from step 6.3) until $k=1$.

6.8) When $k=1$, it must be checked that $b_{1}$ satisfies (11).

6.9) Calculate all $l_{k}$ 's with (9).

6.10) By symmetry the second half of the filter is obtained.

At this point, all the physical dimensions of the waffle-iron filter with transmission zeros at multiple frequencies have been calculated.

\section{DESIGN EXAMPLES}

According to the design methodology proposed above, two different groups of examples will be presented in this Section. The first group will demonstrate the feasibility of designing classical waffle-iron filters with transmission zeros at a single frequency following the novel design technique described in Section II.A. Indeed, three different waffle-iron filters whose transmission zeros are located at three different single frequencies will be designed. The second group includes a novel compact high-power wide-stopband waffle-iron filter with transmission zeros located at different frequencies, following the novel design technique described in Section II.B. Moreover, a prototype of this novel kind of filters will be fabricated and measured.

\section{A. Waffle-iron filter with transmission zeros at one frequency}

Here, three different waffle-iron filters with transmission zeros at a single frequency will be designed with the parameters given in Table I and following the procedure described in Section II.A. The main difference between each filter is the location of the transmission zero. Indeed, the Waffle-Iron \#1 will have a transmission zero further the passband but within the stopband $\left(f_{Z}=25 \mathrm{GHz}\right)$, the transmission zero of the Waffle-Iron \#2 will be around the middle of the stopband $\left(f_{Z}=20 \mathrm{GHz}\right)$, and the Waffle-Iron \#3 will have a transmission zero close to the passband $\left(f_{Z}=15 \mathrm{GHz}\right)$.

The process to obtain the final dimensions of the WaffleIron \#1 is detailed hereinafter. Following Section II.A, the design process begins (Step 1) defining several parameters shown in Table I. We then calculate $\theta_{c}$ by using (5) and the $Z_{i}$ with [20] (Step 2), and the results are shown at the top of Table II. Now, the electrical model calculations to obtain the targeted $\left|S_{11, k}\left(f_{c}\right)\right|$ and $\phi_{k, S_{11}}\left(f_{c}\right)$ using (1) to (5) are done (Step 3), see results at the bottom of Table II. After that, $n_{t}=4, w_{t}=2 \mathrm{~mm}$, $s_{t}=2.072 \mathrm{~mm}$, and $l_{t}=2 \mathrm{~mm}$ are set (Step 4) to achieve the suppression of the higher-order $\mathrm{TE}_{\mathrm{n} 0}$ modes. These values minimize also the excitation of the higher-order modes in the 
inner part of the device and allow an easy manufacturability. Next, $b_{1}$ is set to $5 \mathrm{~mm}$, using (11), to shift the higher-order non$\mathrm{TE}_{\mathrm{n} 0}$ modes (Step 5.1) up to $f_{\max }(30 \mathrm{GHz})$. Then, in order to obtain a transmission zero around $f_{Z}=25 \mathrm{GHz}, b_{k}$ is set following (12) equal to $6 \mathrm{~mm}$ (Step 5.2 and 5.3). Then, for $k=$ 5 (since $\left|S_{11,5}\left(f_{c}\right)\right|$ is the greatest of the $\left|S_{11, k}\left(f_{c}\right)\right|$ values given in Table II) $b_{T 5}$ is computed by (6) employing FEST3D for the EM simulation, which results in $b_{T 5}=2.587$ (Step 5.4). Following (10) $b_{t 5}=0.826 \mathrm{~mm}$ is obtained (Step 5.5). As the $b_{t 5}$ obtained is smaller than the desired one, the value of $b_{5}$ is recalculated by satisfying (6) and $b_{t 5} \geq 1.65 \mathrm{~mm}$ simultaneously (back to Step 5.2 and 5.3). As a result, $b_{5}$, and thus $b_{k}$ for $k=2,3, \ldots,\left(N_{D E}-1\right)$ is fixed to $8.8 \mathrm{~mm}$. Then, utilizing EM simulations, the final dimensions of each DE are separately computed: i) $b_{T k}$ is adjusted using (6) (Step 5.4), and ii) $l_{k, 1}$ and $l_{k, 2}$ are computed by satisfying (7) and (8) (Step 5.6). Finally, $l_{k}$ is computed by (9) (Step 5.7) and a slight adjustment of $l_{k}$ is done. This process has been repeated in all the designed filters, obtaining the final dimensions detailed in Table III.

The comparison between the ideal (electrical model) and the FEST3D simulated frequency response for the synthesized structures before and after the final adjustment is shown in Fig. 4, Fig. 5, and Fig. 6 for the Waffle-Iron \#1, \#2, and \#3

TABLE I. DESIGN PARAMETERS

\begin{tabular}{lc}
\hline \hline All-pole filtering function type & Chebyshev \\
Number of DEs, $N_{D E}$ & 9 \\
Maximum frequency of the passband, $f_{c}$ & $12.65 \mathrm{GHz}$ \\
Maximum attenuation frequency, $f_{0}$ & $22 \mathrm{GHz}$ \\
Maximum frequency of the stopband, $f_{\max }$ & $30 \mathrm{GHz}$ \\
Waveguide width, $a$ & $19.05 \mathrm{~mm}$ \\
Return Loss & $25 \mathrm{~dB}$ \\
\hline \hline
\end{tabular}

TABle II. ElectricAl Model CALCUlations

Elements of the stepped-impedance prototype $Z_{i}$ and $\theta_{c}$

$Z_{0}=Z_{18}=1.000 \quad Z_{1}=Z_{17}=0.702 \quad Z_{2}=Z_{16}=1.681 \quad Z_{3}=Z_{15}=0.450$

$Z_{4}=Z_{14}=2.146 \quad Z_{5}=Z_{13}=0.402 \quad Z_{6}=Z_{12}=2.259 \quad Z_{7}=Z_{11}=0.392$

$Z_{8}=Z_{10}=2.288 \quad Z_{9}=0.390 \quad \theta_{c}=43.39^{\circ}$

\begin{tabular}{ccc}
\hline \multicolumn{3}{c}{ Electrical target performance for each DE } \\
\hline$k$ & $\left|S_{11, k}\left(f_{c}\right)\right|$ & $\phi_{k, S_{11}}\left(f_{c}\right)\left(^{\circ}\right)$ \\
1,9 & 0.438 & 202.11 \\
2,8 & 0.808 & 165.87 \\
3,7 & 0.876 & 157.72 \\
4,6 & 0.888 & 156.27 \\
5 & 0.890 & 155.92 \\
\hline \hline
\end{tabular}

TABLE III. FINAL DiMENSIONS OF EXAMPLES IN SECTION III.A

\begin{tabular}{|c|c|c|c|c|c|c|}
\hline & \multicolumn{2}{|c|}{ Waffle-Iron \#1 } & \multicolumn{2}{|c|}{ Waffle-Iron \#2 } & \multicolumn{2}{|c|}{ Waffle-Iron \#3 } \\
\hline & \multicolumn{2}{|c|}{$\begin{array}{c}b_{1}=5 \mathrm{~mm} \\
b_{k}=8.8 \mathrm{~mm}\end{array}$} & \multicolumn{2}{|c|}{$\begin{array}{c}b_{1}=6 \mathrm{~mm} \\
b_{k}=10.2 \mathrm{~mm}\end{array}$} & \multicolumn{2}{|c|}{$\begin{array}{c}b_{1}=8 \mathrm{~mm} \\
b_{k}=13.35 \mathrm{~mm}\end{array}$} \\
\hline$k$ & $l_{k}(\mathrm{~mm})$ & $b_{T k}(\mathrm{~mm})$ & $l_{k}(\mathrm{~mm})$ & $b_{T k}(\mathrm{~mm})$ & $l_{k}(\mathrm{~mm})$ & $b_{T k}(\mathrm{~mm})$ \\
\hline 1,9 & 2.455 & 0.891 & 2.306 & 1.023 & 2.139 & 1.122 \\
\hline 2,8 & 3.500 & 3.216 & 3.518 & 3.559 & 2.983 & 4.167 \\
\hline 3,7 & 3.558 & 3.500 & 3.421 & 3.909 & 2.805 & 4.638 \\
\hline 4,6 & 3.308 & 3.558 & 3.121 & 3.980 & 2.268 & 4.715 \\
\hline 5 & 3.297 & 3.575 & 3.092 & 4.000 & 2.332 & 4.717 \\
\hline
\end{tabular}

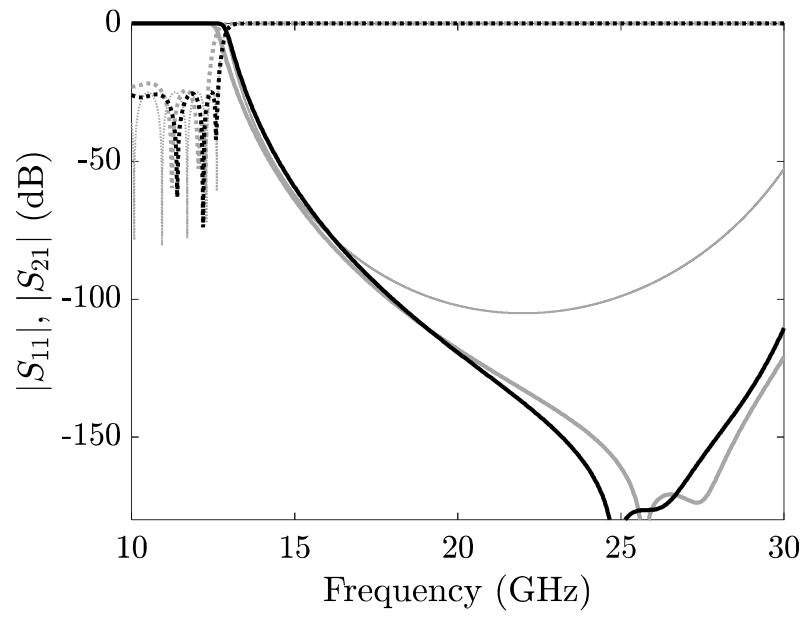

Fig. 4. FEST3D simulated frequency response comparison between the ideal response (grey thin line) and the Waffle-Iron \#1 synthesized with the technique in Section II.A without (grey thick line) and with the final adjustment (black line). $\left|S_{11}\right|$ in dotted line and $\left|S_{21}\right|$ in solid line.

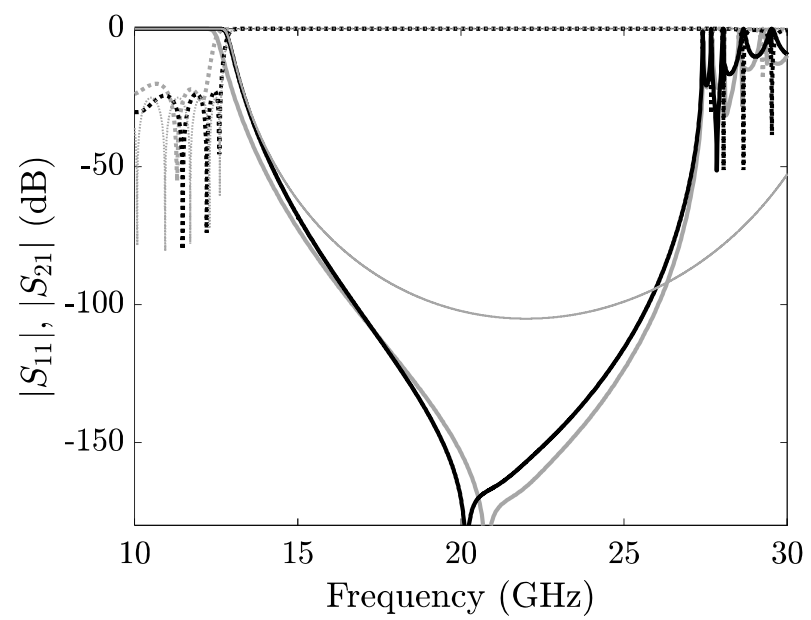

Fig. 5. FEST3D simulated frequency response comparison between the ideal response (grey thin line) and the Waffle-Iron \#2 synthesized with the technique in Section II.A without (grey thick line) and with the final adjustment (black line). $\left|S_{11}\right|$ in dotted line and $\left|S_{21}\right|$ in solid line.

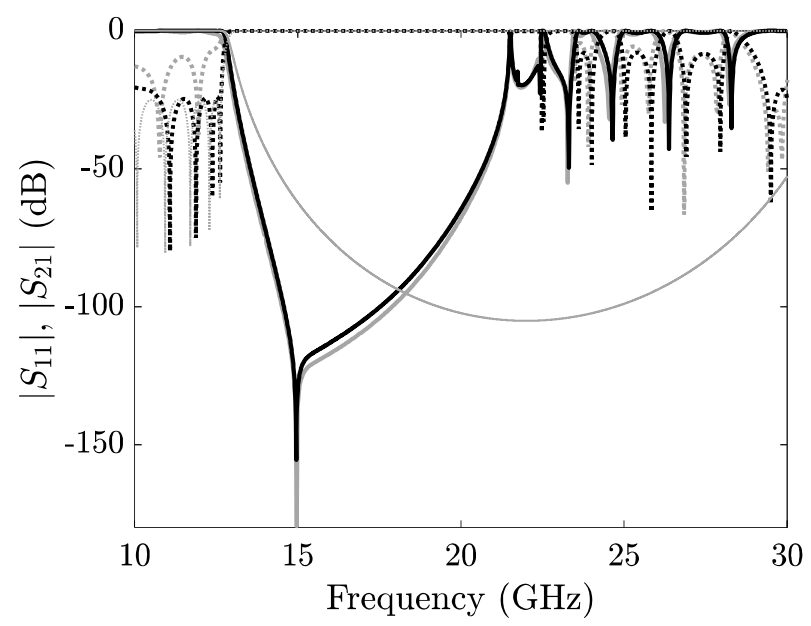

Fig. 6. FEST3D simulated frequency response comparison between the ideal response (grey thin line) and the Waffle-Iron \#3 synthesized with the technique in Section II.A without (grey thick line) and with the final adjustment (black line). $\left|S_{11}\right|$ in dotted line and $\left|S_{21}\right|$ in solid line. 
respectively. As can be observed, the design procedure is more accurate for synthesizing filters that have the transmission zero closer to $f_{\max }$ (or, in other words, less accurate when the transmission zero is close to the passband). The reason being that, if the transmission zero is close to the passband, its associated structure will have higher $b_{k}$ and shorter $l_{k}$, exciting more intensively the higher-order modes which propagate in the waffle-sections and are also evanescently present in the interface of the DEs. As mentioned in Section II, these effects are not directly considered in the design procedure. Even so, the adjustment needed to achieve the final response can be performed in $l_{k}$ only, making the optimization procedure quick and easy for an optimizer module such as the one included in FEST3D. Indeed, the final frequency response for the proposed filters is obtained in a few minutes by using the FEST3D Simplex optimization algorithm tool.

B. Novel Compact High-Power Waffle-iron filter with Transmission Zeros at multiple frequencies

If the stopbands of the previous examples were combined (see Fig. 7), a very steep slope and a wide rejected band might be simultaneously achieved if the different waffle-iron filters were cascaded by means of quarter-wave transformers of several sections as proposed in [19]. However, this is a bulky solution, not at all efficient in terms of mass and volume. Here, a novel compact high-power waffle-iron filter is presented utilizing the design technique proposed in Section II.B and fulfilling the frequency specifications given in Table IV. The designer has several degrees of freedom since, for the targeted specifications to be met, the number and positions of the transmission zeros can be selected, as well as the number of DEs employed to create each zero (multiplicity). In this example, the transmission zeros will be accomplished by a single DE (for the transmission zero closer to the passband) or by two DEs for the rest of transmission zeros (actually, the

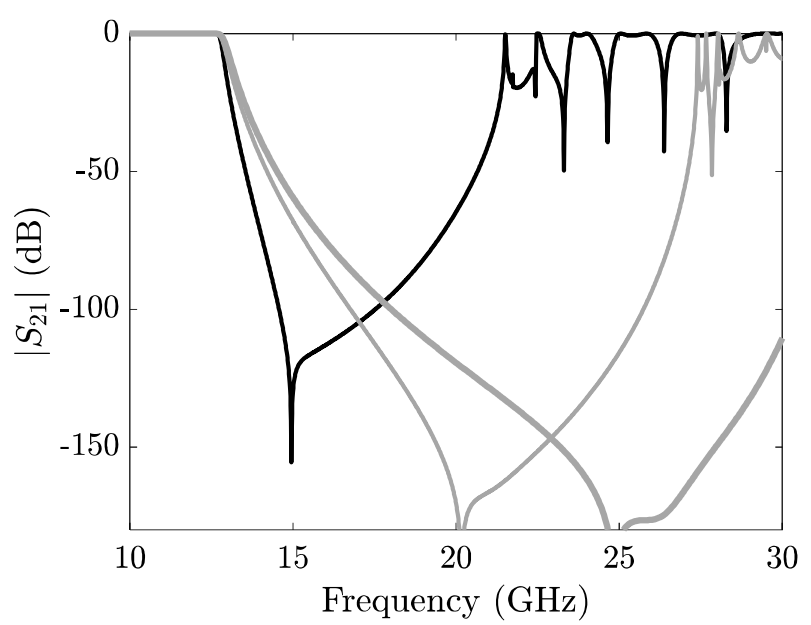

Fig. 7. Staggered stopbands of Waffle-Iron \#1 (grey thick line), Waffle-Iron \#2 (grey thin line), and Waffle-Iron \#3 (black line).

TABLE IV. FREQUENCY SPECIFICATIONS

\begin{tabular}{cccc}
\hline \hline Passband & Return Loss & Stopband & Attenuation \\
\hline $10.7-12.5 \mathrm{GHz}$ & $20 \mathrm{~dB}$ & $14.5-30 \mathrm{GHz}$ & $75 \mathrm{~dB}$ \\
\hline \hline
\end{tabular}

multiplicity is 4 due to the end-to-end symmetry).

The design process begins by selecting the design parameters given in Table I (Step 1) and with the calculations associated to the stepped-impedance prototype given in Table II (Step 2 and 3 ), where a first zero at $15 \mathrm{GHz}$ will be added to fulfil the requirements of Table IV. After that, the physical dimensions of the filter $n_{t}=4, w_{t}=2 \mathrm{~mm}, s_{t}=2.072 \mathrm{~mm}$, and $l_{t}=2 \mathrm{~mm}$ are chosen for the filter to have a good isotropy (Step 4), providing the same features (at a given frequency) for all TEM waves propagating in any direction. This achieves the suppression of the higher-order $\mathrm{TE}_{\mathrm{n} 0}$ modes, minimizes the excitation of the higher-order modes in the inner part of the device, and allows for an easy manufacturability.

Following the design technique described in Section II.B, the design process continues by designing the $k$ th-DE, which will provide the transmission zero closer to the passband (Step 6.1). In this case $k=(9+1) / 2=5$, thus $f_{Z, 5}=15 \mathrm{GHz}$. The baseline value given by (12) is $b_{5}=10 \mathrm{~mm}$ (Step 6.2 and 6.3). Then, $b_{t 5}$ is chosen to be higher than $3 \mathrm{~mm}$ and, utilizing FEST3D to fulfil (6), $b_{5}$ results in $13.35 \mathrm{~mm}$ and $b_{T 5}$ is equal to $4.760 \mathrm{~mm}$ (Step 6.4 and 6.5). By (10) $b_{t 5}=3.83 \mathrm{~mm}$. Moreover, the values of $l_{5,1}$ and $l_{5,2}$ are computed by satisfying (7) and (8) (Step 6.6) and are equal to $1.667 \mathrm{~mm}$ and $0.756 \mathrm{~mm}$ respectively. Then, the out-of-band performance of the 5th-DE is simulated to calculate $f_{M, 5}$, which is around $20 \mathrm{GHz}$ (Step 6.7). Hence, (repeat Step 6.2 to Step 6.7) for $k=4$ $\left(f_{z, 4}=20 \mathrm{GHz}\right)$ the baseline value of $b_{4}=7.5 \mathrm{~mm}$ is obtained using (12). Then, in order to have $b_{t 4}$ higher than $3 \mathrm{~mm}$, and

TABLE V. FinAl DiMENSIONS OF THE NOVEL WAFFLE-IRON FILTER DESIGNED IN SECTION III.B

\begin{tabular}{cccccc}
\hline \hline$k$ & $b_{k}(\mathrm{~mm})$ & $b_{T k}(\mathrm{~mm})$ & $\begin{array}{c}\text { Adjusted } \\
b_{T k}(\mathrm{~mm})\end{array}$ & $l_{k}(\mathrm{~mm})$ & $\begin{array}{c}\text { Adjusted } \\
l_{k}(\mathrm{~mm})\end{array}$ \\
\hline 1,9 & 3.500 & 0.459 & 1.122 & 3.240 & 3.240 \\
2,8 & 7.548 & 2.761 & 3.213 & 3.236 & 2.225 \\
3,7 & 8.800 & 3.392 & 3.388 & 3.776 & 2.883 \\
4,6 & 10.200 & 3.716 & 3.600 & 3.582 & 3.759 \\
5 & 13.350 & 4.760 & 4.752 & 2.233 & 2.200 \\
\hline \hline
\end{tabular}

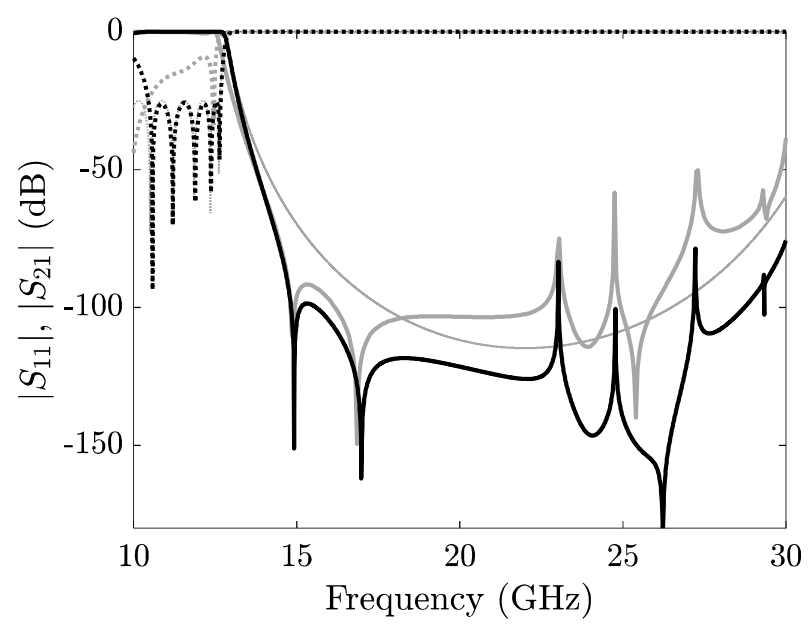

Fig. 8. FEST3D simulated frequency response comparison between the ideal stepped-impedance prototype response (grey thin line) and the novel filter synthesized with the technique in Section II.B without (grey thick line) and with the final adjustment (black line). $\left|\mathrm{S}_{11}\right|$ in dotted line and $\left|\mathrm{S}_{21}\right|$ in solid line. 
satisfying (6), $b_{4}$ is fixed to $10.2 \mathrm{~mm}$ and $b_{T 4}$ is adjusted to $3.716 \mathrm{~mm} . l_{4,1}$ and $l_{4,2}$ are calculated by (7) and (8) and are equal to $1.83 \mathrm{~mm}$ and $1.477 \mathrm{~mm}$ respectively. Then, proceeding iteratively, the physical dimensions of all DEs are computed. The last parameter to be fixed is $b_{1}$, which will be equal to $3.5 \mathrm{~mm}$ to ensure the suppression of the higher-order non- $\mathrm{TE}_{\mathrm{n} 0}$ modes up to $30 \mathrm{GHz}$ (Step 6.8). Finally, $l_{k}$ is computed by (9) (Step 6.9) and the DEs on the right side of the devices will have the same physical parameters (Step 6.10) due to end-to-end symmetry. An adjustment of the dimensions is performed by means of the optimization of only variables $b_{T k}$ and $l_{k}$ using FEST3D with Simplex algorithm, obtaining the final values detailed in Table V.

The frequency response of the synthesized prototype with and without the final adjustment is shown in Fig. 8, along with the ideal response of the electrical prototype. As can be seen in Fig. 8, a very good starting point for the final adjustment is obtained applying the proposed design procedure. In fact, the return loss is already better than $10 \mathrm{~dB}$ for the entire passband

\section{TABLE VI. DESIGN PARAMETERS OF WAFFLE-IRON FILTERS WITHOUT AND WITH TRANSMISSION ZEROS AT ONE FREQUENCY DESIGNED IN SECTION III.B}

\begin{tabular}{lc}
\hline \hline All-pole filtering function type & Chebyshev \\
Maximum frequency of the passband, $f_{c}$ & $12.65 \mathrm{GHz}$ \\
Maximum attenuation frequency, $f_{0}$ & $22 \mathrm{GHz}$ \\
Maximum frequency of the stopband, $f_{\max }$ & $30 \mathrm{GHz}$ \\
Waveguide width, $a$ & $19.05 \mathrm{~mm}$ \\
Return Loss & $25 \mathrm{~dB}$ \\
\hline \hline
\end{tabular}

TABle VII. Electrical MOdel CALCUlations OF WAFFleIRON FILTERS WITHOUT AND WITH TRANSMISSION ZEROS AT ONE FREQUENCY DESIGNED IN SECTION III.B

\begin{tabular}{|c|c|c|c|c|c|}
\hline \multicolumn{3}{|c|}{$\begin{array}{l}\text { Filter without transmission zeros } \\
\qquad N_{D E}=14\end{array}$} & \multicolumn{3}{|c|}{$\begin{array}{l}\text { Filter with transmission zeros at one } \\
\text { frequency } N_{D E}=13\end{array}$} \\
\hline \multicolumn{3}{|c|}{$\begin{array}{l}\text { Elements of the stepped-impedance } \\
\text { prototype } Z_{i} \text { and } \theta_{c}\end{array}$} & \multicolumn{3}{|c|}{$\begin{array}{l}\text { Elements of the stepped-impedance } \\
\text { prototype } Z_{i} \text { and } \theta_{c}\end{array}$} \\
\hline \multicolumn{3}{|c|}{$Z_{0}=Z_{28}=1.000 \quad Z_{1}=Z_{27}=0.699$} & \multicolumn{3}{|c|}{$Z_{0}=Z_{26}=1.000 \quad Z_{1}=Z_{25}=0.699$} \\
\hline \multicolumn{3}{|c|}{$Z_{2}=Z_{26}=1.694 \quad Z_{3}=Z_{25}=0.446$} & \multicolumn{3}{|c|}{$Z_{2}=Z_{24}=1.692 Z_{3}=Z_{23}=0.446$} \\
\hline \multicolumn{3}{|c|}{$Z_{4}=Z_{24}=2.165 \quad Z_{5}=Z_{23}=0.399$} & \multicolumn{3}{|c|}{$Z_{4}=Z_{22}=2.163 \quad Z_{5}=Z_{21}=0.399$} \\
\hline \multicolumn{3}{|c|}{$Z_{6}=Z_{22}=2.282 \quad Z_{7}=Z_{21}=0.388$} & \multicolumn{3}{|c|}{$Z_{6}=Z_{20}=2.279 \quad Z_{7}=Z_{19}=0.388$} \\
\hline \multirow{2}{*}{\multicolumn{3}{|c|}{$\begin{array}{l}Z_{8}=Z_{20}=2.318 \quad Z_{9}=Z_{19}=0.384 \\
Z_{10}=Z_{18}=2.333 Z_{11}=Z_{17}=0.383\end{array}$}} & \multicolumn{3}{|c|}{$Z_{8}=Z_{18}=2.316 \quad Z_{9}=Z_{17}=0.384$} \\
\hline & & & \multicolumn{3}{|c|}{$Z_{10}=Z_{16}=2.330 Z_{11}=Z_{15}=0.383$} \\
\hline \multicolumn{3}{|c|}{$Z_{12}=Z_{16}=2.340 Z_{13}=Z_{15}=0.382$} & \multicolumn{3}{|c|}{$Z_{12}=Z_{14}=2.336 \quad Z_{13}=0.382$} \\
\hline \multicolumn{3}{|c|}{$Z_{14}=2.342 \quad \theta_{c}=43.39^{\circ}$} & \multicolumn{3}{|c|}{$\theta_{c}=43.39^{\circ} \quad---$} \\
\hline \multicolumn{3}{|c|}{$\begin{array}{l}\text { Electrical target performance } \\
\text { for each DE }\end{array}$} & \multicolumn{3}{|c|}{$\begin{array}{l}\text { Electrical target performance } \\
\text { for each DE }\end{array}$} \\
\hline$k$ & $\left|S_{11, k}\left(f_{c}\right)\right|$ & $\phi_{k, S_{11}}\left(f_{c}\right)\left(^{\circ}\right)$ & $k$ & $\left|S_{11, k}\left(f_{c}\right)\right|$ & $\phi_{k, S_{11}}\left(f_{c}\right)\left(^{\circ}\right)$ \\
\hline 1,14 & 0.443 & 202.02 & 1,13 & 0.443 & 202.03 \\
\hline 2,13 & 0.813 & 165.48 & 2,12 & 0.813 & 165.53 \\
\hline 3,12 & 0.880 & 157.39 & 3,11 & 0.880 & 157.42 \\
\hline 4,11 & 0.893 & 155.91 & 4,10 & 0.892 & 155.94 \\
\hline 5,10 & 0.897 & 155.45 & 5,9 & 0.896 & 155.49 \\
\hline 6,9 & 0.898 & 155.27 & 6,8 & 0.898 & 155.31 \\
\hline 7,8 & 0.899 & 155.19 & 7 & 0.898 & 155.25 \\
\hline
\end{tabular}

before any adjustment. The final frequency response of the filter is obtained in a few minutes utilizing the Simplex algorithm of FEST3D. In Fig. 8, the great improvement in the slope between the pass- and the rejected band can also be observed if we compare our novel solution with the classical stepped-impedance prototype. This result should be compared to a classical waffle-iron with and without transmission zeros. Since none of the filters designed in the previous section fulfils the specifications in Table III, new designs are done below.

Let's start designing a classical waffle-iron filter without transmission zeros. Following the technique in [16] and aiming $100 \mathrm{~dB}$ of attenuation at $15 \mathrm{GHz}$ (for a fair comparison), the number of DEs must be increased (from $N_{D E}=9$ in our proposal) to $N_{D E}=14$. The same applies to a waffle-iron filter with transmission zeros at one frequency placed at the middle of the stopband. In this case, the number of DEs must be increased to $N_{D E}=13$. In order to demonstrate this issue, one waffle-iron filter without transmission zeros and one waffleiron filter with transmission zeros at one frequency have been designed following the technique in [16] and Section II.A,

\section{TABLE VIII. FINAL DIMENSIONS OF WAFFLE-IRON FILTERS WITHOUT AND WITH TRANSMISSION ZEROS AT ONE FREQUENCY DESIGNED IN SECTION III.B}

\begin{tabular}{|c|c|c|c|c|c|}
\hline \multirow{3}{*}{\multicolumn{3}{|c|}{$\begin{array}{c}\text { Filter without transmission zeros } \\
\qquad \begin{array}{c}b_{1}=2.25 \mathrm{~mm} \\
b_{k}=4.5 \mathrm{~mm}\end{array} \\
\end{array}$}} & \multirow{3}{*}{\multicolumn{3}{|c|}{$\begin{array}{c}\text { Filter with transmission zeros } \\
\text { at one frequency } \\
b_{1}=5 \mathrm{~mm} \\
b_{k}=8.8 \mathrm{~mm}\end{array}$}} \\
\hline & & & & & \\
\hline & & & & & \\
\hline$k$ & $l_{k}(\mathrm{~mm})$ & $b_{T k}(\mathrm{~mm})$ & $k$ & $l_{k}(\mathrm{~mm})$ & $b_{T k}(\mathrm{~mm})$ \\
\hline 1,14 & 3.083 & 0.456 & 1,13 & 2.439 & 0.905 \\
\hline 2,13 & 3.462 & 1.874 & 2,12 & 3.440 & 3.234 \\
\hline 3,12 & 3.788 & 1.973 & 3,11 & 3.531 & 3.518 \\
\hline 4,11 & 3.616 & 1.993 & 4,10 & 3.261 & 3.578 \\
\hline 5,10 & 3.537 & 1.999 & 5,9 & 3.278 & 3.599 \\
\hline 6,9 & 3.594 & 2.001 & 6,8 & 3.240 & 3.604 \\
\hline 7,8 & 3.552 & 2.002 & 7 & 3.267 & 3.609 \\
\hline
\end{tabular}

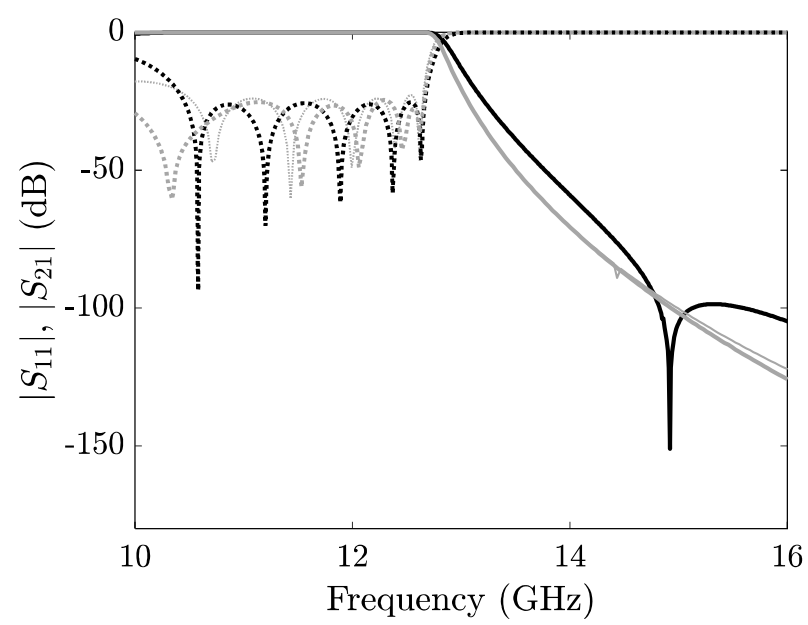

Fig. 9. FEST3D simulated frequency response comparison between the novel filter $N_{D E}=9$ (black line), the waffle-iron filter without transmission zeros $N_{D E}=14$ (grey thin line), and the waffle-iron filter with transmission zeros at one frequency $N_{D E}=13$ (grey thick line). $\left|\mathrm{S}_{11}\right|$ in dotted line and $\left|\mathrm{S}_{21}\right|$ in solid line. 
respectively. The design parameters are given in Table VI, the electrical model characteristic parameters in Table VII, and its final physical dimension in Table VIII.

The comparison between their frequency responses and the one of the novel filter is shown in Fig. 9. If we study the performances of the three filters (see Table IX), the novel filter presents the most compact layout and is the lowest loss solution. Moreover, the novel filter suppresses all higher-order modes up to $30 \mathrm{GHz}$ with a very high attenuation level, as can be observed in Fig. 10.

The high-power behavior (multipactor and corona effects) of the filters designed in this Section has been studied by means of SPARK3D, considering the EM fields previously calculated with CST MWS. The simulations have been done at $12.5 \mathrm{GHz}$ (upper passband edge), which is the frequency where the EM fields are maximum and the multipactor threshold will be minimum. Moreover, the multipactor simulation has been performed using the SEY of silver as background material. As can be seen in Table IX, the multipactor threshold level of the novel filter is quite high compared to the filter without transmission zeros and similar to the waffle-iron filter with transmission zeros at one frequency (as expected). The corona simulation has been conducted considering air at $293{ }^{\circ} \mathrm{K}$ for different pressures as can be seen in Fig. 11, and results are summarized in Table IX.

Finally, two quarter-wave transformers have been added to the input and output ports of the novel filter to reach the WR75

TABLE IX. FEATURES COMPARISON OF THE FILTERS DESIGNED IN SECTION III.B TO FULFIL SPECIFICATION IN TABLE IV

\begin{tabular}{|c|c|c|c|}
\hline & $\begin{array}{c}\text { Filter without } \\
\text { transmission } \\
\text { zeros }\end{array}$ & $\begin{array}{l}\text { Filter with } \\
\text { transmission zeros } \\
\text { at one frequency }\end{array}$ & Novel filter \\
\hline Design method & [16] & Section II.A & Section II.B \\
\hline Size & $74.12 \mathrm{~mm}$ & $67.2 \mathrm{~mm}$ & $40.1 \mathrm{~mm}$ \\
\hline $\begin{array}{l}\text { Insertion loss } \\
\text { (conductivity of Al) }\end{array}$ & $0.22 \mathrm{~dB}$ & $0.19 \mathrm{~dB}$ & $0.15 \mathrm{~dB}$ \\
\hline $\begin{array}{l}\text { Multipactor threshold } \\
\text { (SPARK3D SEY-Ag) }\end{array}$ & $280 \mathrm{~W}$ & $1970 \mathrm{~W}$ & $1855 \mathrm{~W}$ \\
\hline $\begin{array}{l}\text { Corona threshold } \\
\text { (SPARK3D Air-293K) }\end{array}$ & $\begin{array}{c}25 \mathrm{~W} \\
\text { (a) } 18 \mathrm{mbar}\end{array}$ & $\begin{array}{c}62.7 \mathrm{~W} \\
@ 15 \mathrm{mbar}\end{array}$ & $\begin{array}{c}78.6 \mathrm{~W} \\
\text { (a) } 18 \mathrm{mbar}\end{array}$ \\
\hline
\end{tabular}

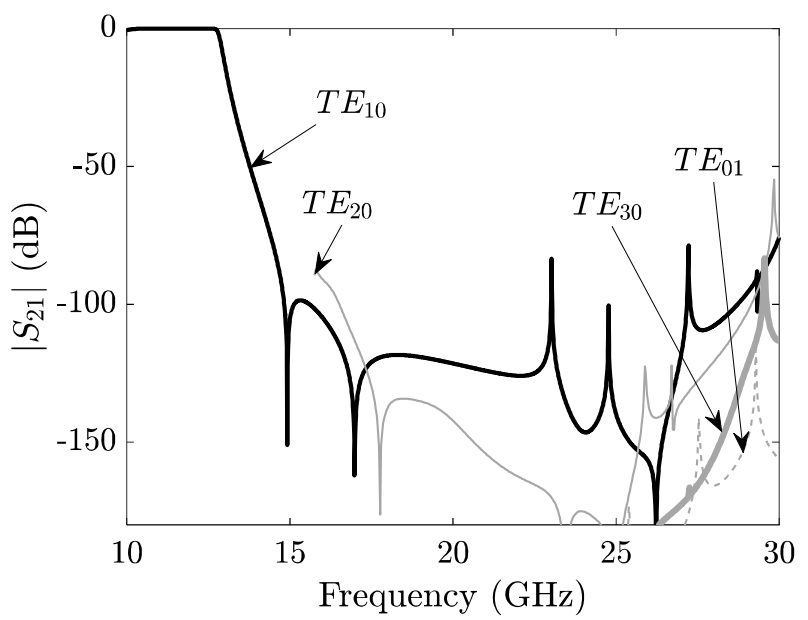

Fig. 10. FEST3D simulated frequency response for the novel filter excited with the fundamental $\mathrm{TE}_{10}$ mode and the higher-order modes: $\mathrm{TE}_{20}, \mathrm{TE}_{30}$, and $\mathrm{TE}_{01}$. standard port dimensions. Then, a prototype of this filter has been fabricated by milling in bare aluminum in two halves cut by the H-plane. The unassembled prototype is photographed in Fig. 12. The frequency response measurement of the prototype has been carried out by an Agilent E8361C PNA Network Analyzer and by means of waveguide-to-coaxial transitions and linear tapers. The measured frequency response of the prototype

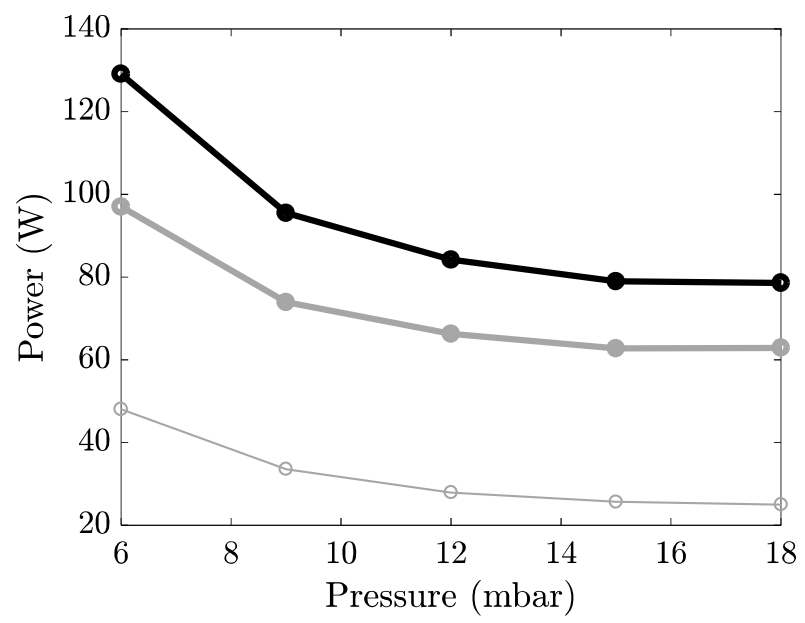

Fig. 11. SPARK3D corona simulation comparison between the novel filter (black line), the waffle-iron filter without transmission zeros (grey thin line), and the waffle-iron filter with transmission zeros at one frequency (grey thick line).

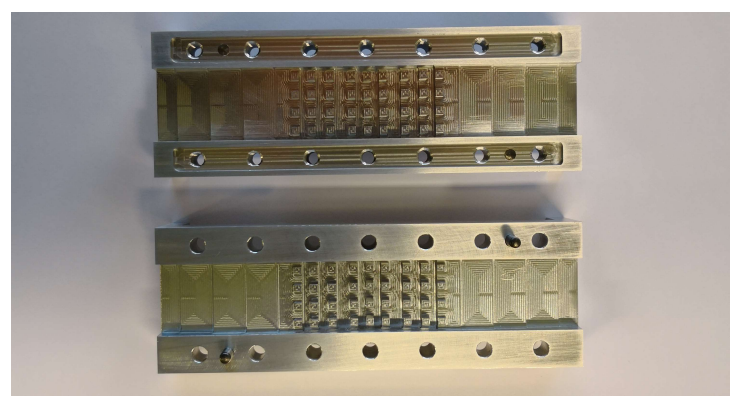

Fig. 12. Unassembled fabricated prototype

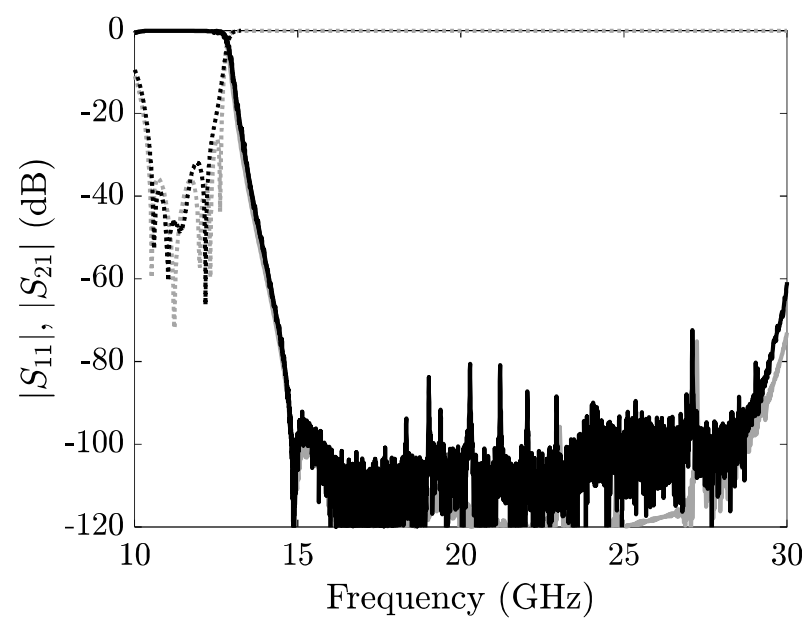

Fig. 13. Frequency response comparison between the measurements of the fabricated prototype (black line) and the CST MWS simulation of the novel filter with transformers and rounded corners. $\left|\mathrm{S}_{11}\right|$ in dotted line and $\left|\mathrm{S}_{21}\right|$ in solid line. 
is shown in Fig. 13 along with the CST MWS simulation of the novel filter considering rounded corners. As can be observed, a remarkable accordance between the measured and simulated results is obtained. The measured insertion loss is better than $0.15 \mathrm{~dB}$ in the filter passband.

\section{CONCLUSION}

In this paper, a novel high-power waffle-iron filter with a wide rejected band and transmission zeros at multiple frequencies in a single compact structure has been presented, including a detailed design procedure. The novel filter is composed of different DEs that produce transmission zeros at different frequencies, achieving a high attenuation over a wide stopband and a steep slope between the passband and the stopband. The filter benefits also from a very compact geometry, keeping the minimum mechanical gap under control. The design procedure is based on mimicking the magnitude and phase responses of the classical stepped-impedance prototype with the set of DEs which compose the novel structure. A stepby-step design technique is described so that it can be easily implemented in a computer and a physical insight is given for all its steps. Moreover, the design method can be also utilized to produce classical waffle-iron filters with transmission zeros at one frequency, surpassing the time-consuming brute-force optimization used nowadays. In order to demonstrate the feasibility of the technique, a novel filter has been designed, simulated, and compared with its classical counterparts. The filter dramatically reduces its size in comparison with the classical solution (the electrical performance of our filter has similar performance to that of a classical filter $68 \%$ larger). Moreover, our design provides lower insertion loss (a reduction of $21 \%$ ), while keeping the high-power performance and the wide rejection bandwidth. A prototype of the novel filter has been fabricated by milling, and the measurement results show an excellent agreement with the simulations. As a final remark, it should be pointed out that the geometry of the teeth does not imply any change in the design procedure. This can be used to enhance even more the power-handling performance of the waffle-iron filters proposed in this paper.

\section{REFERENCES}

[1] S. B. Cohn, "Design relations for the wide-band waveguide filter," in Proceedings of the IRE, vol. 38, no. 7, pp. 799-803, Jul. 1950.

[2] S. B. Cohn, "Analysis of a Wide-Band Waveguide Filter," in Proceedings of the IRE, vol. 37, no. 6, pp. 651-656, June 1949.

[3] M. B. Manuilov, et al., "Full wave hybrid technique for CAD of passive waveguide components with complex cross section," PIERS Online, Vol. 5, 526-530, 2009.

[4] H. Kirino and K. Ogawa, "A $76 \mathrm{GHz}$ multi-layered phased array antenna using a non-metal contact metamaterial waveguide," in IEEE Transactions on Antennas and Propagation, vol. 60, no. 2, pp. 840-853, Feb. 2012.

[5] A. M. K. Saad, "Novel lowpass harmonic filters for satellite application," IEEE MTT-S IMS Digest, San Francisco, CA, 1984, pp. 292-294.

[6] M. Baldelli and D. Maiarelli, "Design and qualification of an X-band output filter with extended harmonics rejection," in 7th International Workshop on Microwave Filters, Noordwijk, The Netherlands, Apr. 2018

[7] P. Soto, et al., "Analysis, design, and experimental verification of microwave filters for safety issues in open-ended waveguide systems," IEEE Transactions on Microwave Theory and Techniques, vol. 48, no.
11, pp. 2133-2140, Nov. 2000.

[8] F. J. Clemente-Fernandez, et al., "Waveguide bandstop filter based on irises and double corrugations for use in industrial microwave ovens," Electronics Letters, vol. 48, no. 13, pp. 772-774, Jun. 2012.

[9] F. Teberio, et al., "Accurate design of corrugated waveguide low-pass filters using exclusively closed-form expressions," 2017 47th Eur. Microw. Conf., Nuremberg, Oct. 2017

[10] L. Matthaei, et al., Microwave Filters, Impedance-Matching Networks, and Coupling Structures, Norwood, MA: Artech House, 1980.

[11] R. E. Collin, Foundations for microwave engineering, Mc Graw-Hill International Editions, 2001.

[12] N. Marcuvitz, Waveguide Handbook. London: Peter Peregrinus, 1986.

[13] R. Levy, "Synthesis of high-power harmonic rejection waveguide filters," 1969 G-MTT IMS, Dallas, TX, 1969, pp. 286-290.

[14] F. Arndt and J. Brandt, "Direct EM based optimization of advanced waffle-iron and rectangular combline filters," IEEE MTT-S International Microwave Symposium, Seattle, WA, 2002, pp. 2053-2056 vol.3.

[15] M. B. Manuilov, K. V. Kobrin, "Field theory CAD of waffle-iron filters," 2005 European Microwave Conference, 2005.

[16] F. Teberio, et al., "Accurate design procedure for waffle-iron low-pass filter," 2018 IEEE MTT-S International Microwave Symposium, Philadelphia, NJ, 2018, pp. 1-4.

[17] S. B. Cohn, "Rounded corners in microwave high-power filters and other components," in IRE Transactions on Microwave Theory and Techniques, vol. 9, no. 5, pp. 389-397, September 1961.

[18] L. Young and B. M. Schiffman, "New and improved types of waffle-iron filters," in Electrical Engineers, Proceedings of the Institution of, vol. 110, no. 7, pp. 1191-1198, July 1963.

[19] E. D. Sharp, "A high-power wide-band waffle-iron filter," in IEEE Transactions on Microwave Theory and Techniques, vol. 11, no. 2, pp. 111-116, Mar 1963

[20] R. J. Cameron, C. M. Kudsia, and R. R. Mansour, Microwave Filters for Communication Systems: Fundamentals, Design and Applications. Hoboken, NJ: John Wiley \& Sons, 2007.

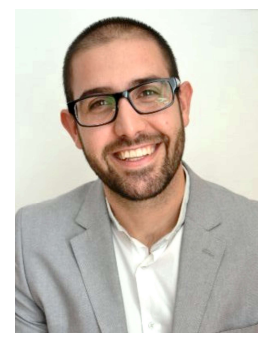

Fernando Teberio received the Telecommunication Engineering, the M.Sc., and Ph.D. degrees from the Public University of Navarre, Spain, in 2009, 2011, and 2018, respectively.

From 2009 to 2018, he was with the Electrical, Electronic, and Communications Engineering Department of the Public University of Navarre. He collaborated in several research projects supported by the Spanish Government, the European Space Agency (ESA) and private companies. Since September 2018, he is working at Alter Technology Group - TÜV Nord. His research interests include periodic structure devices and design and test of passive and active components for communication satellites for microwave and millimetre-wave frequency ranges.

Mr. Teberio was the recipient of a Network Partnering Initiative (NPI) grant from the European Space Agency (ESA) to support his doctoral research.

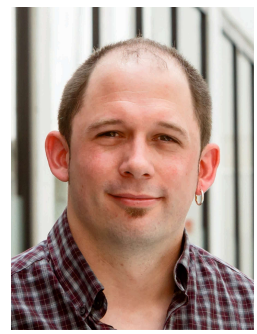

Jon M. Percaz received the Telecommunication Engineering degree and M.Sc degree from the Public University of Navarre, Pamplona, Navarre, Spain, in 2010 and 2015, respectively. He is currently working toward the $\mathrm{PhD}$ degree at the Electrical, Electronic, and Communications Engineering Department of the Public University of Navarre. His research interests include coupled-mode theory and inverse-scattering techniques applied to the synthesis of passive devices in microwave and millimetre wave technologies.

Mr. Percaz is the recipient of a pre-PhD grant from the Public University of Navarra to support his doctoral thesis. 


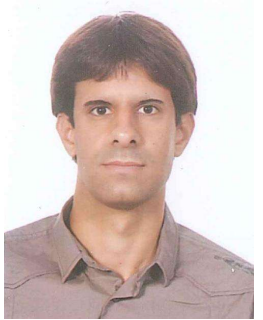

Ivan Arregui (S'08-M'12) received the Telecommunication Engineering, M.Sc., and Ph.D. degrees from the Public University of Navarre, Pamplona, Spain, in 2005, 2008, and 2013, respectively.

$\mathrm{He}$ is currently an Assistant Professor with the Electrical, Electronic, and Communications Engineering Department, Public University of Navarre. He is Co-Founder of the spin-off company TAFCO Metawireless. His current research interests include periodic structure devices for microwave, millimeter-wave and terahertz frequency ranges, numerical techniques for the inverse-scattering synthesis, and the design of passive components for communications satellites.

Dr. Arregui was the recipient of a grant from the Spanish Ministry of Science and Innovation and several prizes including the Junior Research Award of the Public University of Navarre, the HISDESAT prize awarded by the Spanish Telecommunications Engineers Association (COIT/AEIT) for the best Doctoral Dissertation in satellite services, and the Innovation Award Alberto Elzaburu Foundation.

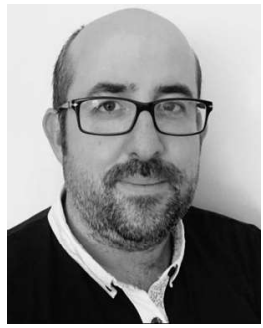

Petronilo Martín-Iglesias (M'12) was born in Caceres, Spain, on April 23, 1980. He received the Telecommunication Engineering degree from the Polytechnic University of Madrid, Madrid, Spain, in 2002, and the Master degree from The University of Leeds, Leeds, U.K., in 2012.

He has been working in industry for over ten years as a Microwave Engineer involved with active (high power amplifiers for radar applications) and passive (filters, multiplexers, couplers, etc.) RF hardware design, including two years as a Radar System Engineer with Indra Sistemas, ISDEFE S.A., and Thales Alenia Space Spain. Since Summer 2012, he has been involved with research and development and project support activities related with RF passive hardware developments for the European Space Agency. His research interests are filter synthesis theory, electromagnetic (EM) design and high-power prediction, as well as advanced manufacturing techniques for RF passive hardware.

Mr. Martín-Iglesias has served as a member of the Technical Program Committee (TPC), IEEE Microwave Theory and Techniques Society (IEEE MTT-S) since 2013.

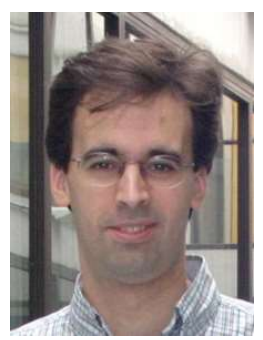

Txema Lopetegi (S'99 - M'03) was born in Pamplona, Navarre, in 1973. He received the M.Sc. and $\mathrm{Ph} . \mathrm{D}$. degrees in telecommunication engineering from the Public University of Navarre, in 1997 and 2002, respectively. Since 1997 he has been with the Electrical, Electronic, and Communications Engineering Department, Public University of Navarre, first as Assistant Professor and since 2006 as Associate Professor. In 1999 and 2000, he was awarded a grant from the Spanish Ministry of Education to support the research in his doctoral thesis. During 2002 and 2003 he was a Research Fellow with the Payload Systems Division, European Space Research and Technology Center (ESTEC), European Space Agency (ESA), at Noordwijk, The Netherlands. His current research interests include metamaterials and synthesized structures in microwave, millimeter wave and $\mathrm{THz}$ technologies, filters and passive components, and coupled mode theory and synthesis techniques using inverse scattering.

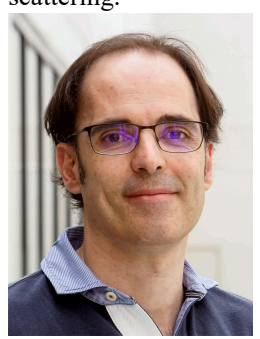

Miguel A. G. Laso (S'99-M'03) received the M.Sc. and Ph.D. degrees in Telecommunications Engineering from the Public University of Navarre (UPNA), Navarre, Spain, in 1997 and 2002, respectively.

From 1998 to 2001, he was with the Electrical, Electronic, and Communications Engineering Department, Public University of Navarre, as a doctoral fellow student. He was an Assistant Professor in the same institution from 2001 to 2006 and an Associate Professor (Profesor Titular) since 2006, always involved with teaching and research duties related to optical communications and microwave engineering. From 2002 to 2003, he was also a Research Fellow with the Payload Systems Division of the European Space Research and Technology Centre, European Space Agency (ESTEC-ESA), Noordwijk, The Netherlands. His current research interests comprise periodic structures, Inverse Scattering problems, and synthesis techniques for filters and multiplexers in the microwave and millimetre-wave frequency ranges, and their applications in wireless and space communications. He is the Head of the Microwave Components Group (MCG) at UPNA and, during his career, he has published dozens of journal papers and contributed to major international conferences. He has also led projects with public and private funding within the MCG-UPNA and he holds several international patents.

Dr. Laso is a member of several professional and scientific international associations including the Institute of Electrical and Electronics Engineers (IEEE), the Optical Society of America (OSA), the International Society for Optics and Photonics (SPIE), and the American Society for Engineering Education (ASEE). He is TPRC member of the MTT-S International Microwave Symposium (IMS) and reviewer of several other international conferences and journals. He was the recipient of a grant from the Spanish Ministry of Education and Science to support his doctoral research at the Public University of Navarre from 1998 to 2001, and another one to support his postdoctoral research at the European Space Agency from 2002 to 2003 . He has been the recipient of several prizes including the Spanish National Prize to the Best Doctoral Dissertation in Telecommunications (2002) awarded by the Spanish Telecommunications Engineers Association (COIT/AEIT) and the Junior Research Award of the Public University of Navarre (2003). He has been also the recipient of the 2005 Spanish National Prize for the Best Project in Innovation in Higher Education awarded by the Spanish Ministry of Education and Science. He was also co-founder of TAFCO MetaWireless S.L., a spin-off company of the Public University of Navarre.

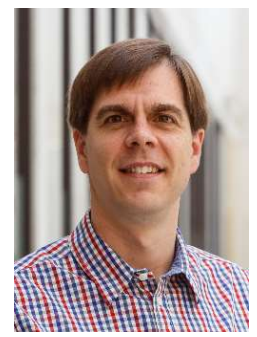

Israel Arnedo (S'05-M'11-SM'17) was born in Tudela, Spain, in 1980. He received the Telecommunication Engineering, M.Sc., and Ph.D. degrees from the Public University of Navarre, (UPNA), Pamplona, Spain, in 2004, 2007, and 2010, respectively.

He is currently an Associate Professor with the Electrical, Electronics and Communications Engineering Department, Public University of Navarre, where he has managed and participated in research projects supported by the Spanish Government, the Natural Sciences and Engineering Research Council of Canada (NSERC), the European Commission, and private companies. Since 2018, he is Vice-dean of the UPNA School of Engineering. His research interests of the microwave, millimeter-wave, and terahertz fields include periodic structure devices, coupled-mode theory, inverse-scattering synthesis, material characterization and their applications in ultra-wideband (UWB) systems, space and satellite technology, biomedical engineering research and industrial products. He has published more than 75 papers in these fields and holds 2 international patents successfully licensed. He is cofounder of the spin-off company TAFCO Metawireless S.L., a spin-off company of the Public University of Navarre. $\mathrm{He}$ is a Reviewer for several international scientific journals.

Dr. Arnedo was the recipient of a Formación de Profesorado Universitario (FPU) grant of the Spanish Ministry of Education and Science to support his doctoral research. In 2012, he was awarded with a José Castillejo grant of the Spanish Ministry of Education to support his postdoctoral research stay at the Institut d'Électronique de Microélectronique et de Nanotechnologie (IEMN), Villeneuve D'Ascq, France. In 2014, he was bestowed the Junior Research Award of the Public University of Navarre for the Best Doctoral Dissertation in the Engineering Field. 